\title{
Analysis of interobserver and intraobserver variation of interpretation of the echocardiographic and Doppler flow determination of cardiac output by the mitral orifice method
}

\author{
GIAN LUIGI NICOLOSI, ERAZEM PUNGERCIC, $†$ EUGENIO CERVESATO, * \\ LIONELLO MODENA, $\dagger$ DOMENICO ZANUTTINI* \\ From the * Servizio di Emodinamica, Ospedale Civile, Via Montereale, Pordenone, and $\dagger$ Servizio di \\ Cardiologia, Soave (Verona), Italy
}

SUMMARY The variability of the interpretation by two individuals of a combined echocardiographic and Doppler method of calculating output was studied in 30 normal adults. In each subject three separate cardiac cycles were recorded to calculate maximal mitral valve orifice, the ratio of mean to maximal mitral valve leaflet separation, and the mean flow velocity through the mitral valve. The recordings were digitised twice by two independent observers. Estimates of cardiac output ranged from 3.2 to $8.11 / \mathrm{min}$. Analysis of variance showed that interobserver and intraobserver variability for these measurements was $5 \cdot 8 \%$ and $6 \cdot 1 \%$ respectively.

It is concluded that the reproducibility for interpreting this non-invasive method is adequate for clinical use in adults with cardiac outputs within the normal range.

A combined echocardiographic and Doppler flow technique that assesses blood flood through the mitral valve has been used to estimate cardiac ouput in animal models ${ }^{12}$ and in patients. ${ }^{34} \mathrm{We}$ have analysed the variability of intraobserver and interobserver results when estimates of flow through the mitral orifice were used to calculate cardiac output in normal adults. ${ }^{1}$

\section{Patients and methods}

We studied thirty four normal adults. Four were subsequently excluded because of poor quality echocardiograms. The mean age of the remaining 30 was 32 (16-52 years) ( 15 men, 15 women).

Echocardiograms and pulsed Doppler recordings were made on a Honeywell Ultra-imager with a $3.5 \mathrm{MHz}$ mechanical transducer. Three prints of stop frame echocardiographic video images of three

Requests for reprints to Dr Gian Luigi Nicolosi, Servizio di Emodinamica, Ospedale Civile, Via Montereale, 33170 Pordenone, Italy.

Accepted for publication 21 January 1986 separate cardiac cycles were used to estimate the maximum mitral valve orifice (from parasternal short axis views).

We obtained $M$ mode recordings of the mitral valve at the same level through the leaflets as the cross sectional study. Again, three cardiac cycles were recorded. Then with the transducer positioned on the cardiac apex a pulsed Doppler sample volume was set to a length of $1 \mathrm{~cm}$ and positioned just below the mitral leaflets. The sample lies parallel to ventricular inflow (or was estimated to be no more than $15^{\circ}$ off parallel). Doppler flow was recorded for three cycles on hard copy at a paper speed of $50 \mathrm{~mm} / \mathrm{s}$. M mode recordings were obtained at the same recording speed.

One investigator obtained all the echocardiographic images and Doppler recordings from resting and haemodynamically stable subjects. On two occasions two observers independently and blindly calculated mitral valve area from hard copies of real time and $\mathrm{M}$ mode traces of the three cycles.

For technical reasons, which were specific to our computer system digitising pad, the Doppler flow curves were traced on to plastic transparency from the hard copy and the transparencies were digitised. 
The densest part of the velocity trace was taken as the modal velocity.

A third observer independently and blindly performed the computer digitisation of the transparency traces of the modal velocities of the three cycles. The computer system calculated the area under each curve to determine the mean velocity of transmitral flow for each cycle.

The maximum mitral area was estimated from tracings on transparencies of the real time stop frame image (both observers produced two tracings for each cycle). The line of the trace followed the inner contour of the mitral leaflets. The transparencies were then digitised independently and blindly by the third observer to calculate maximal mitral valve orifice for the three recorded cycles. Mean to maximal mitral leaflet separation was calculated for the three beats from the $M$ mode traces. The mean mitral maximal area was multiplied by the ratio of the mean to maximal valve leaflet separation to give an effective mean diastolic cross sectional orifice area for the mitral valve (this ratio is said to correct for variations in mitral valve orifice size during diastole). ${ }^{1}$ The two observers traced the anterior and posterior mitral leaflets on the M mode trace. They did this twice for the three recorded cycles. The tracings were digitised blindly by the third observer.

The mean:maximal mitral orifice ratio was calculated from the formula-A/1 $\times h$, where $A$ is the area of the anterior and posterior mitral leaflets on $M$ mode; $h$ is the maximal leaflet separation; and $l$ is the duration of leaflet separation (diastole). Cardiac output was calculated using the formula: $\mathrm{CO}=$ (V CSA 60)/cos $\theta$; where $\mathrm{CO}$ is cardiac output $(\mathrm{l} / \mathrm{min}) ; \mathrm{V}$ is mean velocity throughout the entire cardiac cycle uncorrected for angle $(\mathrm{cm} / \mathrm{s})$, calculated as the mean of the three cycles and of the four measurements performed by the two observers; CSA is effective mean diastolic mitral valve orifice area corrected for diastolic variations $\left(\mathrm{cm}^{2}\right)$, determined by multiplying the cross sectional maximal mitral valve orifice area by the ratio of mean:maximal mitral leaflet separation on the $M$ mode recording. All the measurements were the mean of the three cycles and of the four determinations of the two observers; $\cos \theta$ is the cosine of the angle between the Doppler beam and blood flow.
For the purpose of this study the $\cos \theta$ was assumed to be 1 for all calculations for the following reasons: (a) the purpose of the study was to analyse the interpretative reproducibility and not the effect of the angle $\theta$; (b) in $80 \%$ of our cases the angle was effectively 0 and all other sampling angles were less than $15^{\circ}$; (c) to simplify statistical analysis of the data by avoiding variables not strictly related to interpretative reproducibility; (d) a true correction would have to be performed in three planes and this would be impracticable and subjective.

Final results of cardiac ouput values are given as the mean of the four measurements obtained by the two observers.

\section{STATISTICAL ANALYSIS}

Interobserver and intraobserver interpretative variability was calculated by analysis of variance. The variability was determined not only for cardiac output measurements, but also for each of the three factors included in the formula for calculating cardiac output (cross sectional echocardiographic measurements, $M$ mode echocardiographic measurements, Doppler measurements) and beat to beat for each of the three beats used for the analysis.

The 30 cases were also arbitrarily divided into three groups on the basis of excellent quality recordings (15 cases), good quality recordings (10 cases), and fair quality recordings (five cases) to evaluate the possible influence of the quality of the recording on the final variability.

\section{Results}

Calculated Doppler cardiac output values ranged from $3 \cdot 2$ to $8 \cdot 1 \mathrm{l} / \mathrm{min}$ (mean $5 \cdot 1(1 \cdot 3) 1 / \mathrm{min}$ ). The interobserver and intraobserver variability for cardiac ouput was $0.295 \mathrm{l} / \mathrm{min}(5.8 \%)$ and $0.3101 / \mathrm{min}$ $(6 \cdot 1 \%)$ respectively.

Table 1 shows the interobserver and intraobserver variability for cross sectional measurements, $M$ mode measurements, and Doppler measurements as well as beat to beat variability for the three groups of measurements. Table 2 shows the total interpretative variability for the cross sectional measurements, $M$ mode measurements, and Doppler

Table 1 Interobserver, intraobserver, and beat to beat variability

\begin{tabular}{|c|c|c|c|c|c|c|}
\hline \multirow[t]{2}{*}{ Variability } & \multicolumn{2}{|c|}{ Cross sectional } & \multicolumn{2}{|l|}{$M$ mode } & \multicolumn{2}{|c|}{ Doppler } \\
\hline & $\mathrm{cm}^{2}$ & $\%$ & Units & $\%$ & $\mathrm{~cm} / \mathrm{s}$ & $\%$ \\
\hline $\begin{array}{l}\text { Interobserver } \\
\text { Intraobserver } \\
\text { Beat to beat }\end{array}$ & $\begin{array}{l}0.141 \dagger \\
0.312 \\
0.424 \dagger\end{array}$ & $\begin{array}{l}2 \cdot 5 \\
5 \cdot 5 \\
7 \cdot 5\end{array}$ & $\begin{array}{l}0.0085^{\star} \\
0.0175 \\
0.0147 \dagger\end{array}$ & $\begin{array}{l}(1 \cdot 6) \\
(3 \cdot 3) \\
(2 \cdot 8)\end{array}$ & $\begin{array}{l}0.53 t \\
1.57 \\
1.22 t\end{array}$ & $\begin{array}{l}1 \cdot 8 \\
5 \cdot 5 \\
4 \cdot 3\end{array}$ \\
\hline
\end{tabular}

${ }^{\star} p=0.01$ compared with intraobserver variability.

$\dagger \mathrm{p}=0.001$ compared with intraobserver variability. 
Table 2 Variation in the interpretation of cross sectional, $M$ mode, and Doppler measurements on the basis of the quality of the recordings

\begin{tabular}{|c|c|c|c|c|c|c|}
\hline \multirow[t]{2}{*}{ Quality } & \multicolumn{2}{|c|}{ Cross sectional } & \multicolumn{2}{|l|}{$M$ mode } & \multicolumn{2}{|c|}{ Doppler } \\
\hline & $\mathrm{cm}^{2}$ & $\%$ & Units & $\%$ & $\mathrm{~cm} / \mathrm{s}$ & $\%$ \\
\hline $\begin{array}{l}\text { Excellent }(n=15) \\
\text { Good }(n=10) \\
\text { Fair }(n=5)\end{array}$ & $\begin{array}{l}0.549^{\star} \\
0.677^{\star} \\
0.566^{\star}\end{array}$ & $\begin{array}{r}9.9 \\
11 \cdot 0 \\
10.5\end{array}$ & $\begin{array}{l}0.0245 \\
0.0363 \\
0.0351\end{array}$ & $\begin{array}{l}(4 \cdot 9) \\
(6 \cdot 8) \\
(6 \cdot 0)\end{array}$ & $\begin{array}{l}2 \cdot 36^{\star} \\
2 \cdot 17 \\
2 \cdot 70^{\star}\end{array}$ & $\begin{array}{l}8 \cdot 2 \\
7 \cdot 6 \\
9 \cdot 4\end{array}$ \\
\hline
\end{tabular}

${ }^{\star} \mathrm{p}<0.001$ compared with $\mathrm{M}$ mode (Fisher $\mathrm{F}$ test for variance).

measurements on the basis of the quality of the recordings.

\section{Discussion}

We studied the variability of the interpretation of recorded data not variability due to the recording procedure. Both interobserver and intraobserver variability was close to $6 \%$.

Real time, $\mathbf{M}$ mode, and Doppler measurements were associated with similar degrees of variability, although real time measurements tended to produce more variability and $M$ mode less.

Although adult real time images are inherently less satisfactory than paediatric images, we found that provided the image was suitable for analysis, the difference in their grading was not a major factor in producing the variability (Table 2 ). Although the subjects in our study were clinically normal, image studies were unsatisfactory in four $(12 \%)$ of them. Presumably the proportion of unacceptable recordings in ill patients in intensive care who may have had recent thoracic surgery will be much higher. Our subjects all had cardiac output values in the normal range. Whether or not similar reproducibility would be obtained in low output states has yet to be determined.

We have not entered into the debate on the preferred site for recording blood flow-that is the aorta or transmitral position-nor have we examined the assumption that the mitral orifice is circular in diastole. $^{3}$ We examined the suggestion that variability within and between interpretors can significantly influence the accuracy of measurement. ${ }^{5}$ We found that in a small group of normal individuals the interpretative variability of analysing the echocardiographic and Doppler measurements required for mitral flow calculations was within clinically acceptable limits. A larger series incorporating a range of output states is needed to analyse further factors that may affect variability.

We thank Dr David Sahn, University of California Medical Centre, San Diego for help and advice.

\section{References}

1 Fisher DC, Sahn DJ, Friedman MJ, et al. The mitral valve orifice method for noninvasive two-dimensional echo Doppler determinations of cardiac ouput. Circulation 1983; 67: 872-7.

2 Valdes-Cruz LM, Horowitz S, Mesel E, et al. A pulsed Doppler echocardiographic method for calculation of pulmonary and systemic flow: accuracy in a canine model with ventricular septal defect. Circulation 1983; 68: 597-602.

3 Loeber CP, Goldberg SJ, Allen HD. Doppler echocardiographic comparison of flows distal to the four cardiac valves. $f$ Am Coll Cardiol 1984; 4: 268-72.

4 Lewis JF, Kuo LC, Nelson JC, Limacher MC, Quinones MA. Pulsed Doppler echocardiographic determination of stroke volume and cardiac output: clinical validation of two new methods using the apical window. Circulation 1984; 70: 425-31.

5 Felner JM, Blumenstein BA, Schlant RC, et al. Sources of variability in echocardiographic measurements. Am f Cardiol 1980; 45: 995-1004. 